\title{
Die Matrosenbewegung des Sommers 1917. Zur Erinnerung an Albin Köbis und Max Reichpietsch
}

Angesichts der Frage dieses Sammelbandes nach der "großen Furcht" und Revolutionsangst ist in Bezug auf die Darstellung der Matrosenbewegung des Sommers 1917 in der deutschen Hochseeflotte zu konstatieren, dass sich die Angst der Admiralität und des Marine-Staatssekretariats vor einer Revolution in engen Grenzen hielt. Hier sei kurz auf zwei zentrale Aspekte hingewiesen, die noch ausführlicher erläutert werden: Die Marine-Offiziere glaubten aus einem Gefühl der absoluten gesellschaftlichen Überlegenheit heraus, stets Herr der Lage zu sein und über eine unantastbare Autorität zu verfügen. Sollte diese tatsächlich einmal in Frage gestellt werden, gab es eine rigide Marine-Justiz, die durchgreifen würde. Das zeigte sich im Sommer 1917, als mit Max Reichpietsch und Albin Köbis zwei junge Matrosen zur Abschreckung hingerichtet wurden. Aus der Matrosenbewegung 1917 zogen die Admirale keine Konsequenzen. Sie sahen sich durch ihr hartes Durchgreifen bestätigt, die Ruhe und Ordnung auf den Schiffen wiederhergestellt $\mathrm{zu}$ haben. Aus dieser Annahme heraus beschlossen sie einen letzten Angriffsplan gegen England, der den Untergang der kaiserlichen Marine nach sich gezogen hätte. Hier nahmen sie keinerlei Rücksicht auf die Matrosen, die ihr Leben nicht für die Ehre der Offiziere opfern wollten.

Für das Verständnis der Vorgänge 1917 in der Marine ist ein Blick auf den Aufbau der Flotte, ihre gesellschaftliche Zusammensetzung sowie auf ihre Rolle im Weltkrieg unerlässlich. Die deutsche Kriegsmarine war eine Schöpfung des wilhelminischen Zeitalters und aufs engste mit Kaiser Wilhelm II. verknüpft, der den Anstoß zu einer maritimen Aufrüstung dem Werk Mahans entnahm. ${ }^{1}$ Der

1 Ullrich, Volker: Die nervöse Großmacht. Aufstieg und Untergang des deutschen Kaiserreichs 18711918, Frankfurt am Main 1997, S. 196 und Herwig, Holger H.: Der Einfluß von Alfred Th. Mahan auf die deutsche Seemacht, in: Deutsche Marinen im Wandel. Vom Symbol nationaler Einheit zum Instrument internationaler Sicherheit (Beiträge zur Militärgeschichte, Bd. 63), hrsg. im Auftrag des Militärgeschichtlichen Forschungsamtes von Werner Rahn, München 2005, S. 127-142, hier S. 127-129. 
Kaiser, und mit ihm seine Seeoffiziere, übersah jedoch eine Grundvoraussetzung der Thesen Mahans: Ein Staat könne nicht zugleich als See- und Landmacht Weltgeltung erlangen. ${ }^{2}$ Die Strategie der Hochseeflotte war offensiv ausgerichtet und setzte auf eine Entscheidungsschlacht in der Nordsee. ${ }^{3}$ Da Deutschland durch den Flottenbau die Royal Navy direkt herausforderte, blieb das Verhältnis zu England bis zum Ausbruch des Ersten Weltkrieges belastet. ${ }^{4}$ Der Aufbau einer Flotte war ein finanzielles Risiko, das den deutschen Armeen Mittel entzog und angesichts stetig steigender Kosten den Reichshaushalt auf das schwerste belastete. ${ }^{5}$ Innenpoltisch gab es große Widerstände von der Sozialdemokratie ${ }^{6}$ bis zu den Erzkonservativen, deren prominentester Sprecher der ehemalige Reichskanzler Otto von Bismarck war. ${ }^{7}$

Die neue deutsche Hochseeflotte und das Reichsmarineamt begingen auf dem Gebiet der inneren Führung bereits alle Fehler, die während des Ersten Weltkrieges zu einer Zuspitzung auf den Schiffen bis hin zur Flottenbewegung des Sommers 1917 führten. Die Führung der Flotte lag zu einem ganz überwiegenden Teil in den Händen bürgerlicher Offiziere oder nach der Jahrhundertwende Nobilitierter, denen der Aufstieg in der Armee durch den etablierten preußischen Adel weitgehend versperrt war. ${ }^{8}$ Den Marinevorgesetzten gelang es nicht, von Ausnah-

2 Herwig, Mahan, S. 137-138 und Rahn, Werner: Strategische Optionen und Erfahrungen der deutschen Marineführung 1914 bis 1944: Zu den Chancen und Grenzen einer mitteleuropäischen Kontinentalmacht gegen Seemächte, in: Deutsche Marinen im Wandel. Vom Symbol nationaler Einheit zum Instrument internationaler Sicherheit (Beiträge zur Militärgeschichte, Bd.63), hrsg. im Auftrag des Militärgeschichtlichen Forschungsamtes von Werner Rahn, München 2005, S. 197-234, hier S. 200.

3 Nägler, Frank: Operative und strategische Vorstellungen der Kaiserlichen Marine vor dem Ersten Weltkrieg, in: Skagerrakschlacht. Vorgeschichte - Ereignis - Verarbeitung (Beiträge zur Militärgeschichte, Bd. 66), im Auftrag des Militärgeschichtlichen Forschungsamtes hrsg. von Michael Epkenhans/ Jörg Hillmann/ Frank Nägler, München 2009, S. 19-56, hier S. 22 und Herwig, Mahan, S. 132.

4 Willms, Johannes: Nationalismus ohne Nation. Deutsche Geschichte von 1789 bis 1914 (Fischer Taschenbuch, Bd. 4350), Frankfurt am Main 1985, S. 655 und Ullmann, Hans-Peter: Das Deutsche Kaiserreich 1871-1918 (Neue historische Bibliothek, Edition Suhrkamp, Bd. $1546=$ N.F. 546), Frankfurt am Main 1995, S. 216-217.

5 Wehler, Hans-Ulrich: Deutsche Gesellschaftsgeschichte, Bd. 3: Von der "Deutschen Doppelrevolution« bis zum Beginn des Ersten Weltkrieges 1849-1914, München 1995, S. 1136. Siehe auch Berghahn, Volker R.: Der Tirpitz-Plan. Genesis und Verfall einerinnenpolitischen Krisenstrategie unter Wilhelm II. (Geschichtliche Studien zu Politik und Gesellschaft, Bd. 1), Düsseldorf 1971, S. 618.

6 Bergien, Rüdiger: Flotte und Medien im Kaiserreich, in: Deutsche Marinen im Wandel. Vom Symbol nationaler Einheit zum Instrument internationaler Sicherheit (Beiträge zur Militärgeschichte, hrsg. vom Militärgeschichtlichen Forschungsamt, Bd. 63), hrsg. im Auftrag des Militärgeschichtlichen Forschungsamtes von Werner Rahn, München 2005, S. 143 -160, hier S. 151.

7 Förster, Stig: Der doppelte Militarismus. Die deutsche Heeresrüstungspolitik zwischen Status-QuoSicherung und Aggression 1890 und 1913 (Veröffentlichungen des Instituts für europäische Geschichte Mainz, Bd. 118), Stuttgart 1985, S. 77 und Persius, Lothar: Wie es kam daß der Anstoß zur Revolution von der Flotte ausging, Berlin 1919, S. 4.

8 Wehler, Hans-Ulrich: Das Deutsche Kaiserreich 1871-1918 (Kleine Vandenhoeck-Reihe, Bd. 1380), Göttingen 1983, S. 167. 
men abgesehen, ein spannungsfreies Verhältnis mit den unteren Dienstgraden aufzubauen. ${ }^{9}$

Bereits mit dem Kriegsausbruch im August 1914 rächten sich die beiden entscheidenden Fehler. Die Royal Navy war an einer Entscheidungsschlacht nicht interessiert und nutzte ihre strategische Überlegenheit aus, indem sie eine Fernblockade Deutschlands am Ärmelkanal und im Norden Schottlands bevorzugte. ${ }^{10}$ Die Hochseeflotte hätte unter diesen Gegebenheiten nur eine äußerst riskante Offensive fern der eigenen Basis beginnen können. Um unkalkulierbare Verluste zu vermeiden, entschieden sich der Kaiser und Admiralsstabschef von Pohl gegen eine große Schlacht. ${ }^{11}$

In dieser Konstellation kam es zu kleineren Schlachten, die stets zugunsten der überlegenen Royal Navy ausgingen. In dem Seegefecht am 28. August 1914 vor Helgoland verlor die deutsche Flotte drei Kreuzer, ${ }^{12}$ das Doggerbank-Gefecht vom 23. Januar 1915 führte zum Untergang der "Blücher«. ${ }^{13}$ Trotz des unglücklichen Ausgangs bewährte sich die Mannschaft und leistete sogar hervorragende Arbeit, als es gelang, die schwer angeschlagene "Seydlitz» wieder in den Heimathafen zu bringen. ${ }^{14}$

Danach war die Hochseeflotte weitgehend zur Untätigkeit verurteilt, was wiederum zu starken Spannungen an Bord der Schiffe führte. Zu Beginn des Jahres 1915 verließen die wenigen altgedienten Seeoffiziere, die mehrheitlich ein gutes Verhältnis zur Besatzung hatten, die Schiffe, um andere Funktionen, vornehmlich in U-Booten, zu übernehmen. An ihre Stelle rückten sehr junge Offiziere, die sich eher durch "Schneid" als durch Leistung auszeichneten. ${ }^{15}$

Die Matrosen reagierten auf diese Entwicklung. Angesichts der gravierenden personellen Veränderungen fanden sie sich zusammen und besprachen ihre Situation. ${ }^{16}$

In dieser bereits angespannten Lage kam es am 31. Mai 1916 zur SkagerrakSchlacht. An dem Seegefecht waren insgesamt 250 Schiffe mit rund 100000 Mann

9 Wehler, Deutsche Gesellschaftsgeschichte, Bd. 3, S. 1132 und Howard, Michael: Kurze Geschichte des Ersten Weltkrieges, München 2004, S. 19.

10 Strachan, Hew: Der Erste Weltkrieg. Eine neue illustrierte Geschichte, München 2006, S. 246.

11 Rahn, Werner: Strategische Probleme der deutschen Seekriegführung 1914-1918, in: Der Erste Weltkrieg. Wirkung, Wahrnehmung, Analyse (Serie Piper, Bd. 1927), im Auftrag des Militärgeschichtlichen Forschungsamtes hrsg. von Wolfgang Michalka, München 1994, S. 346 und Ullmann, Das Deutsche Kaiserreich, S. 341-365, hier S. 232.

12 Epkenhans, Michael: Die kaiserliche Marine im Ersten Weltkrieg: Weltmacht oder Untergang? In: Der Erste Weltkrieg. Wirkung, Wahrnehmung, Analyse (Serie Piper, Bd. 1927), im Auftrag des Militärgeschichtlichen Forschungsamtes hrsg. von Wolfgang Michalka, München 1994, S. 319-340, hier S. 323. 13 Strachan, Der Erste Weltkrieg, S. 252.

14 Scheer, Admiral Reinhard: Deutschlands Hochseeflotte im Weltkrieg, Berlin 1920, S. 135 - 136.

15 Vgl. die Tagebucheinträge Stumpfs. Werk des Untersuchungsausschusses der Verfassunggebenden Nationalversammlung (fortan: WUA), Bd. 10/I, S. 45.

16 WUA, Bd. 9/II, S. 243. 
Besatzung beteiligt. ${ }^{17}$ Die Royal Navy verlor drei Schlachtkreuzer und elf weitere Schiffe. ${ }^{18} 6.784$ britische Matrosen starben oder waren verwundet. Elf deutsche Schiffe wurden versenkt, ${ }^{19} 3.058$ deutsche Matrosen waren tot oder verwundet. ${ }^{20}$ Auch wenn die Hochseeflotte als Sieger aus der Seeschlacht hervorging, war allen Verantwortlichen klar, dass weitere Gefechte in dieser Größenordnung angesichts der zahlenmäßigen englischen Überlegenheit in kurzer Zeit den Untergang der deutschen Flotte bedeuteten. ${ }^{21}$

Die Besatzungen der Hochseeflotte verhielten sich in dieser Schlacht mustergültig. Die vorhandenen Spannungen zwischen Mannschaften und Offizieren wurden ausgeblendet. ${ }^{22}$ Durch zahlreiche hohe militärische Auszeichnungen würdigte die Marineleitung insbesondere die Leistungen der Matrosen. ${ }^{23}$ Albin Köbis an Bord der "Prinzregent Luitpold» bewährte sich als Heizer ebenso wie Max Reichpietsch als Signalgeber der "Friedrich der Große». ${ }^{24}$

Der kurzfristigen Euphorie über den Sieg folgte schnell wieder der alltägliche Dienst mit seinen zahlreichen Problemen. Es zeichnete sich ab, dass die Skagerrak-Schlacht für lange Zeit die letzte große militärische Aktion der Marine während des Krieges bleiben würde ${ }^{25}$ und somit ein wichtiges Ventil fehlte, angestauten Unmut abzubauen. Bereits im Herbst 1916 kam es zu ersten Gehorsamsverweigerungen auf der »Helgoland «,26 denen sich ein öffentlicher Protest durch das Aushängen eines Transparentes auf der »Posen« Ende des Jahres 1916 anschloss. Die Matrosen bemängelten ausdrücklich die schlechte Behandlung und monierten, dass sie nur der "Pöbel» für die Vorgesetzten seien. ${ }^{27}$ Im

17 Auf deutscher Seite standen nach Schmiedel 45000 Mann, auf englischer Seite rund 60000 Matrosen. Schmiedel, Karl: Der U-Boot-Krieg und die Schlacht am Skagerrak, in: Deutschland im ersten Weltkrieg, Bd. 2: Januar 1915 bis Oktober 1917, hrsg. von Willibald Gutsche, Berlin 1970, S. 329-335, hier S. 334 .

18 Die "von der Tann« versenkte die »Indefatigable», die "Lützow» versenkte das Schlachtschiff »Invincible» und die "Queen Mary» sank unter dem Geschützfeuer der »Seydlitz«. Rehder, Jacob: Die Verluste der Kriegsflotten 1914-1918, neu erarbeitet und erweitert von Helmut Sander, München 1969, S. $76-77$.

19 Die beiden Schiffe »Lützow» und "Pommern» gingen am 1. Juni 1916 unter. Rehder, Verluste der Kriegsflotten, S. 24-25.

20 Die Zahl der Verwundeten und Toten nach Strachan, Der Erste Weltkrieg, S. 261. Schmiedel, UBoot-Krieg und Skagerrak, S. 334 gibt die Zahl der Gefallenen mit 6.094 britischen und 2.551 deutschen Seeleuten an. Die geringere Zahl an getöteten oder verletzten Matrosen lag auch an der sichereren Bauweise der deutschen Schiffe. Otto, Helmut/ Schmiedel, Karl: Der erste Weltkrieg. Militärhistorischer Abriß (Schriften des Militärgeschichtlichen Instituts der DDR, Kleine Militärgeschichte), 4. Aufl., Berlin 1983, S. 220.

21 Rahn, Strategische Probleme, S. 353 und Strachan, Der Erste Weltkrieg, S. 261.

22 WUA, Bd. 9/II, S. 54- 55.

23 Ebd., S. 105.

24 In der Stammrolle Max Reichpietschs wurde die Teilnahme an der Skagerrak-Schlacht ausdrücklich vermerkt. Bundesarchiv-Militärarchiv Freiburg (fortan: BA-MA), RM 20-112, Rechtsverhältnisse, Auszug aus der Stammrolle des Matrosen Max Reichpietsch, Bl. 127-130.

25 Rahn, Strategische Probleme, S. 353.

26 WUA, Bd. 10/II, S. 193.

27 WUA, Bd. 9/I, S. 18-19. 
Frühjahr 1917 nahm der militärische Drill noch weiter zu, ${ }^{28}$ Beleidigungen und Beschimpfungen waren alltäglich ${ }^{29}$ und wurden so gut wie nie geahndet ${ }^{30}$. Der "Steckrübenwinter« 1916/17 sorgte für eine katastrophale Versorgungslage bei den Matrosen ${ }^{31}$ und der Zivilbevölkerung ${ }^{32}$, während die Verpflegung der Offiziere weiterhin gut blieb. ${ }^{33}$ Diese Ungleichheit verstärkte die Unzufriedenheit der Matrosen ganz erheblich. ${ }^{34}$ Doch die Marineleitung ignorierte das Problem hartnäckig. 35

Anfang des Jahres 1917 forderten einzelne Matrosen, sich gemeinsam gegen ihre unwürdige Lage aufzulehnen. ${ }^{36}$ Im Mai kam es zu ersten Zusammenkünften der Matrosen um Willi Sachse, Willi Weber und Max Reichpietsch. ${ }^{37} \mathrm{Zu}$ ihnen kamen als weitere führende Köpfe der Matrosenbewegung des Sommers 1917 Albin Köbis und Hans Beckers hinzu. Die fünf Matrosen waren sich schnell einig in der Beurteilung ihrer Lage auf den Schiffen, hatten aber in der Folge oft deutliche Meinungsverschiedenheiten darüber, wie eine wirkungsvolle Organisation aufgebaut werden konnte. Die unterschiedlichen persönlichen Hintergründe der Beteiligten erschwerten immer wieder ein einheitliches Vorgehen.

Max Reichpietsch wurde religiös erzogen, ging in die Lehre und kam noch zu Friedenszeiten zur Marine. ${ }^{38}$ Nach ersten problemlosen Jahren kam es zu Konflikten mit den Vorgesetzten, die mit einer sechsmonatigen Haftstrafe endeten. Nach der Verbüßung bewährte sich Max Reichpietsch in verantwortungsvoller Position. ${ }^{39}$ Während des Sommers setzte er große Hoffnungen auf die Unabhängige So-

\footnotetext{
28 WUA, Bd. 9/II, S. 296.

29 Beckers, Hans: Wie ich zum Tode verurteilt wurde. Die Marinetragödie im Sommer 1917 (FischerTaschenbücher, Bd. 5967), Frankfurt am Main 1986, S. 17.

30 WUA, Bd. 9/I, S. 17.

31 Horn, Daniel: The German Naval Mutinies of World War I, New Brunswick 1969, S. 98.

32 Regulski, Christoph: Klippfisch und Steckrüben. Die Lebensmittelversorgung der Einwohner Frankfurts am Main im Ersten Weltkrieg 1914-1918. Eine Studie zur deutschen Wirtschafts- und Innenpolitik in Kriegszeiten (Studien zur Frankfurter Geschichte, Bd. 60), Wiesbaden/ Frankfurt a. M. 2012, S. 317. 33 Siehe die anschauliche Darstellung bei Beckers, Zum Tode verurteilt, S. 18-19.

34 BA-MA Freiburg, RM 47-140, Gerichtsangelegenheiten 1917-1918, Aussage Fritz Höscheler in der Voruntersuchungssache Luise Zietz, Berlin, 19. November 1917, Bl. 162.

35 Deist, Wilhelm (Bearb.): Militär und Innenpolitik im Weltkrieg 1914-1918 (Quellen zur Geschichte des Parlamentarismus und der politischen Parteien, Reihe 2, Bd. 1 und 2), hrsg. im Auftrage der Kommission für Geschichte des Parlamentarismus und der politischen Parteien und des Militärgeschichtlichen Forschungsamtes von Erich Matthias/ Hans Meier-Welcker, Düsseldorf 1970, hier Bd. 2, Dokument Nr. 383, S. 1037-1038. Der Marineleitung und den Offizieren an Deck fehlte jegliches Verständnis für die Lage der Matrosen. Wolz, Nicolas: Das lange Warten. Kriegserfahrungen deutscher und britischer Seeoffiziere 1914 bis 1918 (Zeitalter der Weltkriege, Bd. 3), Paderborn u. a. 2008, S. 249.

36 Der Oberheizer Rebe forderte in einem Brief vom 6. Februar 1917, »der Anarchie von oben muss die Revolution entgegen gestellt werden.«WUA, Bd. 9/I, S. 459-460.

37 Bernhard, Hans-Joachim: Der Aufstand in der deutschen Hochseeflotte im Sommer 1917, Diss. Leipzig 1958, S. 15.

38 Dittmann, Wilhelm: Die Marine-Justizmorde von 1917 und die Admiralsrebellion von 1918, Berlin 1926, S. 32.

39 BA-MA Freiburg, RM 20-112, Rechtsverhältnisse, Auszug aus der Stammrolle des Matrosen Max Reichpietsch, B1. 127-130.
} 
zialdemokratische Partei Deutschlands (USPD). Albin Köbis kam als gelernter Mechaniker zur Marine und versah seinen Dienst als Heizer. Politisch links stehend setzte er sich vermehrt mit den Gedanken des Sozialismus auseinander und gelangte zu einer Philosophie des Anarchismus. ${ }^{40}$ Der konkreten politischen Arbeit in einer Partei stand er ablehnend gegenüber. ${ }^{41}$ Dabei vertrat Köbis seine Ansichten stets sehr geradlinig und aufrichtig.42 Eine vergleichbare politisch-gesellschaftliche Grundlage erarbeitete sich auch Hans Beckers. Aus einer christlichen Bergarbeiterfamilie stammend fiel er früh vom Glauben ab, da für ihn die Heilige Schrift und das Leben der Menschen nicht vereinbar waren. ${ }^{43}$ Beckers kam zur Freien Sozialistischen Jugendbewegung und interessierte sich für die anarchistischen Schriften Max Stirners. ${ }^{44}$ Unter den maßgebenden Persönlichkeiten der Matrosenbewegung lehnte er eine Beteiligung der USPD am nachdrücklichsten ab.

Willi Sachse war innerhalb der Bewegung der politisch am besten geschulte Matrose. Als gelernter Mechaniker trat er früh der Sozialdemokratischen Jugendbewegung bei ${ }^{45}$ und kannte die Klassiker des Sozialismus in wichtigen Textstellen auswendig. ${ }^{46}$ Er war sehr begabt und intelligent, aber auch sehr eigen mit einem Hang zum Psychopathischen. Über Willi Weber ist nur sehr wenig bekannt. Er kam 1912 zur Marine ${ }^{47}$ und hatte sich nach eigenen Angaben zuvor nicht politisch betätigt. ${ }^{48}$ Im Prozess gegen ihn bekannte sich Weber zur Mehrheits-Sozialdemokratie. ${ }^{49}$

Die unterschiedlichen Perspektiven führten oft zu intensiven Gesprächen über das Vorgehen in der Bewegung und erschwerten es, zügig eine einheitliche Linie zu finden. Seit den ersten Treffen im Mai 1917 tauschten die Matrosen ihre Erfahrungen aus und unterhielten sich über sozialistische Lektüren ${ }^{50}$ wie die "Spartakusbriefe ${ }^{51}$. Auch die russische Revolution hinterließ einen tiefen Eindruck bei den deutschen Matrosen, ${ }^{52}$ da sie in ihr das Vorbild einer Veränderung politischer Verhältnisse sahen. Mit der Gründung der USPD am 6. April 1917 in Gotha be-

40 Horn, Naval Mutinies, S. 102.

41 Beckers, Zum Tode verurteilt, S. 62.

42 Horn, Naval Mutinies, S. 102.

43 Beckers, Zum Tode verurteilt, S. 77.

44 WUA, Bd. 9/II, S. 257.

45 Ebd., S. 225.

46 Horn, Naval Mutinies, S. 78.

47 WUA, Bd. 10/I, S. 323.

48 WUA, Bd. 9/II, S. 289.

49 Ebd., S. 231.

50 BA-MA Freiburg, RM 47-140, Gerichtsangelegenheiten 1917-1918, Aussage Willi Richard Sachse in der Voruntersuchungssache Luise Zietz, Berlin, 10. November 1917, Bl. 26.

51 Bundesarchiv Berlin (fortan: BA Berlin), SgY30/1942, Nachlass Walter Beling, Bl. 9. Vgl. auch Kaulisch, Revolutionäre Antikriegsbewegung, S. 701.

52 Kaulisch, Baldur: Das Übergreifen der revolutionären Antikriegsbewegung auf Heer und Flotte, in: Deutschland im Ersten Weltkrieg, Bd. 2: Januar 1915 bis Oktober 1917, hrsg. von Willibald Gutsche, Berlin 1968, S. $695-708$, hier S. 695. 
stand in Deutschland eine Partei, die sich für einen schnell zu schließenden Frieden ohne Annexionen und Kontributionen aussprach. ${ }^{53}$ Auf diese Entwicklungen reagierte die Marineleitung mit der Einführung des „Vaterländischen Unterrichts «, der seine beabsichtigte Wirkung jedoch völlig verfehlte. ${ }^{54}$

Die Matrosen fanden sich auf der "Friedrich der Große» zu ersten Vertrauensmännerwahlen zusammen, ${ }^{55}$ Max Reichpietsch übernahm bald eine führende Position. ${ }^{56}$ Es war damit eine erste organisatorische Grundlage geschaffen, auf der die Matrosen den Entschluss fassten, sich gegen die miserable Verpflegung zu wehren $^{57}$, sodass sich die Marineleitung gezwungen sah zu handeln. Sie genehmigte Anfang Juli 1917 Menagekommissionen, die den Matrosen eine Mitsprache bei der Verpflegung einräumten. ${ }^{58}$ Gegen den Willen der Schiffskommandanten ${ }^{59}$ setzten die Matrosen die Wahlen zur Menagekommission durch. ${ }^{60}$ Mit dem Einblick in die Bücher hatten sie nun eine Kontrollmöglichkeit über die Einkäufe. ${ }^{61}$ Das Essen wurde tatsächlich besser, ${ }^{62}$ wenngleich eine Verpflegung wie die der Offiziere nie erreicht werden konnte. ${ }^{63}$ Die psychologische Wirkung dieser Änderungen ist dabei nicht zu unterschätzen: Die Matrosen konnten den Offizieren erstmals offiziell legitimiert gegenübertreten, was ihr Selbstbewusstsein förderte. ${ }^{64}$ Darüber hinaus gelang es durch die Kommissionen, die in Anfängen bereits bestehende politische Arbeit zu vertiefen. ${ }^{65}$

Ganz entscheidend zur Entwicklung der Matrosenbewegung des Sommers 1917 trug der Kontakt einzelner führender Matrosen zur USPD bei, die sich für einen raschen Friedensschluss einsetzte und auf der bevorstehenden Stockholmer Konferenz der sozialistischen Parteien vertreten sein würde. Um den Krieg schnellstmöglich zu beenden, erwogen die Matrosen eine organisierte Gehorsamsverweigerung als Unterstützung für die zu erwartenden politischen Beschlüsse. Das

53 Krause, Hartfrid: USPD. Zur Geschichte der Unabhängigen Sozialdemokratischen Partei Deutschlands (Studien zur Gesellschaftstheorie), Frankfurt a. M./ Köln 1975, S. 90.

54 Bernhard, Hans-Joachim: Die Entstehung einer revolutionären Friedensbewegung in der deutschen Hochseeflotte im Jahre 1917, in: Revolutionäre Ereignisse und Probleme in Deutschland während der Periode der Großen Sozialistischen Oktoberrevolution 1917/1918. Beiträge zum 40. Jahrestag der Grossen Sozialistischen Oktoberrevolution (Schriften des Instituts für Geschichte, Reihe I, Bd. 6), hrsg. vom Institut für Geschichte an der Deutschen Akademie der Wissenschaften zu Berlin unter der Red. von Albert Schreiner, Berlin 1957, S. 91-140, hier S. 109.

55 Bernhard, Aufstand der Hochseeflotte, S. 18 und 49.

56 Dittmann, Marine-Justizmorde, S. 11.

57 WUA, Bd. 9/I, S. 4 und BA-MA Freiburg, RM 47-140, Gerichtsangelegenheiten 1917-1918, Aussage

Willi Richard Sachse in der Voruntersuchungssache Luise Zietz, Berlin, 11. November 1917, Bl. 30.

58 WUA, Bd. 9/II, S. 263.

59 BA Berlin, SgY30/1204, Nachlass Otto Liedloff, Bl. 4.

60 WUA, Bd. 10/I, S. 48.

61 Bernhard, Aufstand in der Hochseeflotte, S. 66.

62 WUA, Bd. 10/I, S. 48.

63 BA Berlin, SgY30/0262, Nachlass Ernst General, Bl. 5.

64 Dieser Effekt ist in der historischen Forschung noch nicht ausdrücklich erwähnt worden.

65 BA Berlin, SgY30/1942, Nachlass Walter Beling, Bl. 10. 
grundlegende Missverständnis bestand allerdings darin, wie dieses Ziel erreicht werden konnte. Während die Matrosen den passiven Widerstand in Form der Gehorsamsverweigerung als das Mittel ihrer Bewegung auserkoren, wussten die erfahrenen Parlamentarier in Deutschland um die Gefährlichkeit dieses Vorgehens, das mit sehr drastischen Strafen geahndet werden würde.

Das erste und auch wichtigste Treffen zwischen Max Reichpietsch, Wilhelm Dittmann und Luise Zietz fand während Reichpietschs Urlaub nach dem 12. Juni 1917 statt. ${ }^{66}$ Max Reichpietsch erklärte die wachsende Unzufriedenheit mit der schlechten Versorgung sowie mit der oftmals unangebrachten Behandlung durch die Offiziere.67 Das Verbot sozialistischer Lektüre durch die Marineleitung beschäftigte die Matrosen ebenfalls sehr intensiv. ${ }^{68}$ Ein wesentliches Gesprächsthema bildete die Besetzung der von Staatssekretär von Capelle genehmigten Menagekommissionen.69 Max Reichpietsch betonte die politische Orientierung vieler Matrosen an den Zielen der USPD und ihren Wunsch, Mitglied der Partei zu werden. Für die politische Bildung auf den Schiffen wünschten sich seine Kameraden Informationsmaterial. ${ }^{70}$

Wilhelm Dittmann reagierte zurückhaltend. Er war erfreut über die Zustimmung innerhalb der Hochseeflotte zur Politik der USPD und erklärte sich gerne bereit, legale, von der Zensur genehmigte Broschüren zur Verfügung zu stellen, ${ }^{71}$ warnte aber eindringlich vor politischen Versammlungen. ${ }^{72}$ Bereits Gespräche über politische Themen konnten aus Sicht des erfahrenen Abgeordneten für die Matrosen gefährlich werden. ${ }^{73}$ Eine Parteimitgliedschaft war nach Dittmann möglich, aber nicht entscheidend. Wichtig war die Zustimmung durch die Mannschaften der Marine und weniger eine formelle Zugehörigkeit. ${ }^{74}$ Für Wilhelm Dittmann und Luise Zietz war die ruhige, besonnene Förderung der politischen Einstellung der Matrosen das zentrale Anliegen. Konkrete Schritte der Matrosen,

66 WUA, Bd. 9/I, S. 211. Zum Zeitpunkt des Urlaubs siehe auch Deist, Militär und Innenpolitik, Bd. 2, Besprechung von Capelle mit Helfferich, Dokument 382, S. 1026 und Dittmann, Marine-Justizmorde, S. 31.

67 WUA, Bd. 9/I, S. 35 - 36. Auch BA-MA Freiburg, RM 47-140, Gerichtsangelegenheiten 1917-1918, Aussage Willi Richard Sachse in der Voruntersuchungssache Luise Zietz, Berlin, 10. November 1917, Bl. 27.

68 BA-MA Freiburg, RM 47-140, Gerichtsangelegenheiten 1917-1918, Aussage Ewald Vogtherr in der Voruntersuchungssache Luise Zietz, Cöpenick, 8. November 1917, B1. 22.

69 Dittmann, Marine-Justizmorde, S. 31.

70 RM 47-140, Gerichtsangelegenheiten 1917-1918, Aussage Wilhelm Dittmann in der Voruntersuchungssache Luise Zietz, Berlin, 14. November 1917, Bl. 142.

71 Dittmann, Marine-Justizmorde, S. 36.

72 Bernhard, Revolutionäre Friedensbewegung, S. 115 - 116 und WUA, Bd. 9/I, S. 200 - 203.

73 Dittmann, Marine-Justizmorde, S. 34.

74 WUA, Bd. 9/I, S. 37. 
wie Gehorsamsverweigerung oder aktiver Widerstand, wurden nicht erwähnt und waren aus Sicht der USPD ausgeschlossen. ${ }^{75}$

In einem zweiten kurzen Treffen im Reichstag, bei dem Ewald Vogtherr und Hugo Haase anwesend waren, ging es in erster Linie um den Bachmann-Befehl zum Verbot sozialistischer Lektüre, der aus Sicht des Marineexperten der USPD Vogtherr rechtswidrig war. ${ }^{76}$ Alle USPD-Abgeordneten warnten Max Reichpietsch ausdrücklich vor politischen Versammlungen, die den Matrosen sehr schnell gefährlich werden würden. ${ }^{77} \mathrm{Um}$ die Matrosen in ihrem politischen Eifer zu dämpfen, verwiesen die Abgeordneten auf die bevorstehende Konferenz in Stockholm, deren Ergebnisse man erst einmal abwarten sollte. ${ }^{78}$ Aber nicht nur für die Matrosen waren politische Schritte gefährlich. Der USPD war klar, dass aufgedeckte Organisationen von Matrosen, die Mitglieder ihrer Partei waren, umgehend Untersuchungen gegen die USPD zur Folge haben würden.

So waren die zurückhaltenden Beurteilungen durch die USPD auch für die weiteren Besucher Albin Köbis, Paul Calmus und Willi Sachse eher ernüchternd als aufmunternd. Besonders Willi Sachse hob hervor, dass sowohl er selbst als auch seine Kameraden von der Haltung der USPD enttäuscht waren. ${ }^{79}$ Wenn der Kontakt zur USPD für die weitere Entwicklung der Matrosenbewegung dennoch von entscheidender Bedeutung war, so lag das in erster Linie an der Persönlichkeit Max Reichpietschs und seiner Interpretation der Treffen. Er wähnte die USPD grundsätzlich hinter den Zielen der Matrosen und glaubte trotz der Warnungen an die Unterstützung durch die Partei bei weiteren politischen und organisatorischen Schritten. ${ }^{80}$

Die lokale Zusammenarbeit zwischen den Matrosen und der USPD gestaltete sich in Kiel schwierig. Die dortigen USPD-Mitglieder Güth und Sens standen einer politischen Verbindung mit den Matrosen zurückhaltend gegenüber. ${ }^{81}$ Zwischen Sens und Reichpietsch kam es am 14. Juli 1917 zu einer heftigen Auseinandersetzung, als Sens erklärte, Arbeiterstreiks während des Krieges seien vertretbar, keineswegs aber militärische. ${ }^{82}$ Reichpietsch hielt sie durchaus für gerechtfertigt

75 BA-MA Freiburg, RM 47-140, Gerichtsangelegenheiten 1917-1918, Aussage Wilhelm Dittmann in der Voruntersuchungssache Luise Zietz, Berlin, 14. November 1917, Bl. 142.

76 WUA, Bd. 9/I, S. 213.

77 BA-MA Freiburg, RM 47-140, Gerichtsangelegenheiten 1917-1918, Aussage Hugo Haase in der Voruntersuchungssache Luise Zietz, Cöpenick, 12. November 1917, Bl. 19.

78 Bernhard, Revolutionäre Friedensbewegung, S. 119.

79 WUA, Bd. 9/I, S. 301 und WUA, Bd. 9/II, S. 247.

80 Bernhard, Revolutionäre Friedensbewegung, S. 118-119.

81 WUA, Bd. 9/I, S. 56 und WUA, Bd. 9/II, S. 205-206. Auch Bernhard, Revolutionäre Friedensbewegung, S. 131.

82 BA-MA Freiburg, RM 47-140, Gerichtsangelegenheiten 1917-1918, Aussage Wilhelm Sens in der Voruntersuchungssache Luise Zietz, Berlin, 20. November 1917, Bl. 156. 
und wähnte die USPD hinter sich. ${ }^{83}$ Die Anwendung von Gewalt schied aber für die Matrosen und die USPD grundsätzlich aus. ${ }^{84}$

Nach den Treffen zwischen Matrosen und USPD-Abgeordneten standen im Juli 1917 zwei Entwicklungen innerhalb der Bewegung im Vordergrund. Zum einen wehrten sich die Matrosen vermehrt gegen schlechte Behandlung und verdorbenes Essen, zum anderen versuchten sie, ihre Interessen in einer Organisation zu bündeln. Anfang Juli 1917 fanden sich die Matrosen Willi Sachse, Max Reichpietsch, Albin Köbis und Fischer ${ }^{85}$ von der "Pillau» erstmals schiffsübergreifend in Kiel zusammen. Aus dieser konstituierenden Versammlung ging der Soldatenbund hervor. ${ }^{86}$ Einigkeit bestand darin, sich vermehrt gegen die Missstände an Bord zu wehren. ${ }^{87}$ Kontrovers diskutierten die Matrosen eine eventuelle Anlehnung an die USPD. Während Beckers strikt dagegen war, sprach sich Reichpietsch erfolgreich für eine Orientierung an der Partei aus. ${ }^{88}$ Beckers formulierte das Ziel, Ende August oder Anfang September eine organisierte Gehorsamsverweigerung durchzuführen. ${ }^{89}$

Die Nachricht von der Gründung des Soldatenbundes verbreitete sich schnell unter den Matrosen. Hans Beckers und Albin Köbis hatten die Aufgabe, Kontakte mit anderen Schiffen zu vertiefen. ${ }^{90}$ Ein erster Schritt des Bundes bestand in der gezielten Unterschriftensammlung für die Stockholmer Konferenz. Allein im IV. Geschwader kamen 5.000 Unterschriften für die Friedenspolitik der USPD zusammen, die dem Wert der Listen allerdings skeptisch gegenüberstand. ${ }^{91}$ Um die geplante Gehorsamsverweigerung erfolgreich durchführen zu können, knüpften die Matrosen Kontakte zum I. Geschwader. Ein Treffen am 25. Juli zwischen den Besatzungen der "Friedrich der Große» und der "Rheinland " gab der Bewegung entscheidende Impulse. Auch dort organisierten sich die Mannschaften gegen Ende Juli 1917.92

Am 20. Juli kam es auf der "Pillau» zu einem ersten Ausmarsch von Matrosen. Sie entfernten sich während der Dienstzeit unerlaubt vom Schiff, kamen aber

83 BA-MA Freiburg, RM 47-140, Gerichtsangelegenheiten 1917-1918, Aussage Willi Richard Sachse in der Voruntersuchungssache Luise Zietz, Berlin, 17. November 1917, B1. 106.

84 BA-MA Freiburg, RM 47-140, Gerichtsangelegenheiten 1917-1918, Aussage Reuter an Bord der "Ostfriesland», 28. August 1917, B1. 120.

85 Sachse sah in Fischer den eher unpolitischen, gewaltsamen Agitator, der die Matrosen nur wenig überzeugen konnte. Der Vorname Fischers ist in WUA nicht überliefert. WUA, Bd. 9/II, S. 253.

86 Beckers, Zum Tode verurteilt, S. 20.

87 Dittmann, Marine-Justizmorde, S. 7 und Bernhard, Aufstand in der Hochseeflotte, S. 58.

88 WUA, Bd. 9/II, S. 272 und Horn, Naval Mutinies, S. 103 und S. 126.

89 Beckers, Zum Tode verurteilt, S. 20.

90 Bernhard, Aufstand in der Hochseeflotte, S. 63 und Horn, Naval Mutinies, S. 100.

91 BA-MA Freiburg, RM 47-140, Gerichtsangelegenheiten 1917-1918, Aussage Hugo Haase in der Voruntersuchungssache Luise Zietz, Cöpenick, 12. November 1917, B1. 20.

92 Bernhard, Aufstand in der Hochseeflotte, S. 92 - 94. 
nach kurzer Zeit zurück. ${ }^{93}$ Die Bestrafung fiel sehr milde aus. ${ }^{94}$ Aufgrund des schlechten Essens verließen Mannschaften der "Bremen« Mitte Juli 1917 ihr Schiff für einige Stunden. ${ }^{95}$ Auf der "König Albert» kam der Kapitän des Schiffes unter ungeklärten Umständen ums Leben. Aufgrund eines vorhergegangenen heftigen Disputs zwischen Schiffsleitung und Matrosen ${ }^{96}$ kam die Möglichkeit in Betracht, ein Matrose habe den angetrunkenen Kapitän Torbeke über Bord geworfen. Beweise konnte die eingesetzte Untersuchungskommission aber nicht erbringen. ${ }^{97}$ Dieser Vorfall, der immerhin die bewusste Tötung eines Offiziers möglich erscheinen ließ, war kein Grund für die militärische Führung, ihr Handeln zu überdenken. Alle Anzeichen eines Umdenkens blieben aus, von Furcht oder gar Angst war nichts zu merken.

Parallel zu den ersten Aktionen arbeitete Max Reichpietsch in führender Position daran, die Matrosenbewegung zu stärken. Er besaß umfassende Kontakte zu anderen Schiffen und die besten Verbindungen zur USPD. ${ }^{98}$ Besonders erfolgreich war Reichpietsch in der Beschaffung von Informationsmaterial zur politischen Bildung. ${ }^{99}$ Eine weitere wichtige Persönlichkeit des Soldatenbundes war der Leipziger Redakteur Alfred Herre. Er wirkte mäßigend auf die Matrosen ein, hob aber die Bedeutung der Unterschriftensammlung hervor. Den Matrosen war nach Herres Rede klar, dass die Anwendung von Gewalt ihren sicheren Tod bedeutete. ${ }^{100}$ Sie mussten den Weg der Gehorsamsverweigerung beschreiten, um ihr Ziel eines baldigen Friedensschlusses zu erreichen. Damit begann nach Beckers die organisierte Friedensaktion in der Hochseeflotte. ${ }^{101}$

Als in Folge dieser Versammlung die Flottenbewegung immer stärker anwuchs, trafen sich ihre herausragenden Vertreter um den 23. Juli 1917 im Wilhelmshavener Lokal »Tivoli«, um sich schiffsübergreifend in der Flottenzentrale zu organisieren. Auch bestanden noch grundsätzliche Meinungsverschiedenheiten über die Bedeutung der USPD für die Matrosenbewegung, ${ }^{102}$ während die Anwendung von Gewalt einhellig abgelehnt wurde. ${ }^{103}$ Die Matrosen verfolgten das Konzept ei-

\footnotetext{
93 WUA, Bd. 9/II, S. 39.

94 Beckers, Zum Tode verurteilt, S. 39.

95 WUA, Bd. 9/II, S. 44.

96 BA Berlin, SgY30/1204, Nachlass Otto Liedloff, B1. 8.

97 WUA, Bd. 9/I, S. 68.

98 Ebd., S. 243.

99 WUA, Bd. 10/I, S. 50.

100 BA-MA Freiburg, RM 47-140, Gerichtsangelegenheiten 1917-1918, Antwort Hugo Haase an den Untersuchungsrichter des Reichsgerichts, Dr. Holthöfer, in der Voruntersuchungssache Luise Zietz, Berlin, 13. November 1917, Bl. 145.

101 BA-MA Freiburg, RM 47-140, Gerichtsangelegenheiten 1917-1918, Aussage Johann Beckers in der Voruntersuchungssache Luise Zietz, Cöpenick, 11. Dezember 1917, B1. 98. Auch WUA, Bd. 9/II, S. 270.

102 Beckers, Zum Tode verurteilt, S. 61-62.

103 BA-MA Freiburg, RM 47-140, Gerichtsangelegenheiten 1917-1918, Aussage Willi Richard Sachse in der Voruntersuchungssache Luise Zietz, Berlin, 25. November 1917, Bl. 109.
} 
ner evolutionären Entwicklung auf Grundlage der Gruppenentwicklung auf den einzelnen Schiffen. ${ }^{104}$ Die neu begründete Flottenzentrale war eine erforderliche Organisation für die geplante Gehorsamsverweigerung und blieb ohne Satzung oder Statut. ${ }^{105}$ Sie wurde von den führenden Persönlichkeiten auf den Schiffen getragen, wobei Max Reichpietsch im Zentrum der Bewegung stand.106 Doch lag ihr Schwerpunkt weiterhin auf den einzelnen Schiffen, ${ }^{107}$ die autonom blieben. ${ }^{108}$

Am 1. August wurde offensichtlich, dass der Soldatenbund und die Flottenzentrale noch ganz am Anfang standen. An diesem Tag verließen 49 Matrosen der »Prinzregent Luitpold" das Schiff, nachdem tags zuvor statt einer Freiwache militärischer Dienst angesetzt worden war. Sie kehrten nach wenigen Stunden zurück. ${ }^{109}$ Der Kommandant verhängte harte Arreststrafen und ließ den aus seiner Sicht verantwortlichen Heizer Bernhard Spanderen verhaften. ${ }^{110}$ Die Vertrauensmänner und die Mitglieder der Menagekommission berieten sich am Abend des 1. Augusts mit ihren Kameraden von Schiffen des I. Geschwaders, wie sie der willkürlichen Bestrafung begegnen sollten. ${ }^{111}$ Sie beschlossen einen Protestausflug für den nächsten Tag und sicherten sich gegenseitig ihre Solidarität zu. ${ }^{12}$ Albin Köbis war klar, dass durch die spontan erfolgte Entfernung der Prinzregenter die Pläne des Soldatenbundes, gegen Ende August eine abgesprochene Gehorsamsverweigerung durchzuführen, hinfällig wurden. ${ }^{113}$

Am Morgen des 2. Augusts entfernten sich 600 Mann der „Prinzregent Luitpold" von ihrem Schiff ${ }^{114}$ und marschierten den Deich entlang nach Rüstersiel. Dort hielten sie eine Versammlung ab, in der Albin Köbis einen baldigen Frieden forderte. Ein angeblicher Aufruf, gewaltsam die Fesseln zu sprengen, kann anhand der Quellenlage nicht nachgewiesen werden. ${ }^{115}$ Der weitere friedliche Ver-

104 BA-MA Freiburg, RM 47-140, Gerichtsangelegenheiten 1917-1918, Aussage Johann Beckers in der Voruntersuchungssache Luise Zietz, Cöpenick, 11. Dezember 1917, Bl. 99.

105 WUA, Bd. 9/II, S. 320.

106 Ebd., S. 268 und BA-MA Freiburg, RM 47-140, Gerichtsangelegenheiten 1917-1918, Aussage Johann Beckers in der Voruntersuchungssache Luise Zietz, Cöpenick, 11. Dezember 1917, Bl. 100.

107 Horn, Naval Mutinies, S. 131.

108 Beckers sprach der Zentrale später Kompetenzen ab: „Sachse, Weber und Reichpietsch fühlten sich als Herrscher in der Zentrale, aber hatten nichts zu sagen."Siehe für das Zitat WUA, Bd. 9/II, S. 272.

109 Deist, Militär und Innenpolitik, Bd. II, Kommando der Hochseestreitkräfte an Chefs der Immediatbehörde der Marine vom 4. August 1917, Dokument Nr. 374, S. 996.

110 WUA, Bd. 10/I, Urteil in der Untersuchungssache gegen Handgrödinger und Mitangeklagte, S. 317. 111 Die Matrosen kamen von den Schiffen »Friedrich der Große», »Kaiser», "Kaiserin» und »Pillau». Siehe dazu WUA, Bd. 9/II, S. 276.

112 BA Berlin, SgY 30/1204, Nachlass Otto Liedloff, Bl. 6 und Beckers, Zum Tode verurteilt, S. 40.

113 WUA, Bd. 9/II, S. 324.

114 Nach Abzählen der Anwesenden ergab sich die Zahl von 600 Matrosen. Siehe dazu Beckers, Zum Tode verurteilt, S. 41. Liedloff erwähnte 1.000 Soldaten, wobei er die Zahl aus der Erinnerung schätzte. Siehe dazu BA Berlin, SgY 30/1204, Nachlass Otto Liedloff, Bl. 7.

115 BA Berlin, R 43/1395 m, Reichskanzlei, Parteien/Sozialdemokraten, Beschwerde Max Reichpietschs gegen das Urteil, Vernehmung gemäß $§ 423$ Militärstrafgesetzbuch, Wilhelmshaven, 26. August 1917, Bl. 239 und WUA, Bd. 9/II, S. 309. 
lauf des Protestes und der geordnete Rückmarsch sprechen eindeutig für den passiven Widerstand der Matrosen. ${ }^{116}$

Zurück an Bord besprach der zufällig anwesende Admiral von Mauve mit den zurückgekehrten Matrosen den Vorfall und ordnete die Freilassung der tags zuvor Verhafteten an. ${ }^{117}$ Nach diesem scheinbaren Sieg der Matrosen verließ das Schiff unter Belagerungszustand den Hafen. Zuvor schrieb Hans Beckers einen Zettel für die Kameraden, der sie an ihre zugesagte Solidarität erinnerte. ${ }^{118}$

Noch am 2. August setzte auf den Schiffen eine umfassende Untersuchung der Vorkommnisse ein. Schnell waren die ersten Matrosen verhaftet, belastendes Material konnte sichergestellt werden. ${ }^{119}$ Die zugesagten Solidaritätsaktionen waren unmöglich geworden, Gewalt lehnten die Matrosen auch in dieser zugespitzten Situation ab. ${ }^{120}$ Die Marineleitung bewertete den Vorfall als nicht weiter schwerwiegend. Admiral von Müller notierte nach dem Ausmarsch, dass »leichte Meutereien « stattgefunden hätten. ${ }^{121}$ Andere Offiziere sprachen von einem "Dummerjungenstreich «122.

Die Bewegung war durch diesen Schlag ganz entscheidend geschwächt. Dennoch war sie unter den Matrosen bereits so weit gefestigt, dass es noch weitere Aktionen gab, die aber durch den verstärkten Einsatz von Spitzeln zügig aufgedeckt wurden.

Auf der "Westfalen« versuchten die Matrosen am 7. August 1917, die Bewegung fortzuführen und mit der USPD in Kontakt zu treten, um das weitere Vorgehen zu besprechen. ${ }^{123}$ Hier zeigt sich der Einfluss Max Reichpietschs, der stets beteuerte, die USPD stünde hinter ihnen. Nach einem organisierten Streik beim Kohleladen griff die Justiz hart durch. Die Strafen fielen mit Zuchthaus für 39 Matrosen drakonisch aus. ${ }^{124}$ Die Matrosenorganisation der "Westfalen" erhielt mit der Verhaftung Paul Brügges einen entscheidenden Rückschlag. Ein letztes Treffen zwischen Matrosen der »Westfalen« und der »Ostfriesland» konnte durch

116 Beckers, Zum Tode verurteilt, S. $42-43$.

117 Bernhard, Aufstand in der Hochseeflotte, S. 119 und Horn, Naval Mutinies, S. 96.

118 WUA, Bd. 9/II, S. 280 sowie Beckers, Zum Tode verurteilt, S. 43 - 44 und Kielmansegg, Peter Graf: Deutschland und der Erste Weltkrieg (Athenaion-Bibliothek der Geschichte), Frankfurt a.M. 1968, S. 381.

119 WUA, Bd. 9/II, S. 249.

120 Ebd., S. 249.

121 Görlitz, Walter (Hrsg.): Regierte der Kaiser? Kriegstagebücher, Aufzeichnungen und Briefe des Chefs des Marine-Kabinetts Admiral Georg Alexander von Müller 1914-1918, Göttingen 1959, S. 311. 122 WUA, Bd. 10/I, S. 135.

123 Neu, Heinrich: Die revolutionäre Bewegung auf der deutschen Flotte 1917-1918 (Beiträge zur Geschichte der nachbismarckischen Zeit und des Weltkrieges, Bd. 10), hrsg. von Fritz Kern, Stuttgart 1930, S. 36.

124 Bernhard, Aufstand in der Hochseeflotte, S. 147. 
die Tätigkeit des Spitzels Adams aufgedeckt werden. ${ }^{125}$ Mit den Verhaftungen des 2. Septembers 1917 war die Matrosenbewegung endgültig zerschlagen.

Den am 2. August festgenommenen Matrosen war bewusst, gegen die militärische Ordnung verstoßen zu haben. ${ }^{126}$ Dass es aber dann sehr schnell um Leben oder Tod ging, hätte keiner der Verhafteten erwartet. ${ }^{127}$

Zuerst waren die Verhöre noch recht allgemein und besaßen keinerlei bedrohlichen Charakter. ${ }^{228}$ Das änderte sich aber grundlegend, als unter Leitung von Marinehilfskriegsgerichtsrat Dr. Dobring führende Matrosen verhört wurden. Er begrüßte die inhaftierten Männer als "Todeskandidaten « ${ }^{129}$, richtete mehrfach eine Waffe auf sie ${ }^{130}$ und dehnte die sich rasch wiederholenden Verhöre auf viele Stunden aus ${ }^{131}$. Seine Absicht war es, auf der Grundlage inzwischen gefundener Belege die Verantwortung der USPD für die Entwicklung in der Flotte nachzuweisen. ${ }^{132}$ Dieses Ziel erreichte Dr. Dobring durch die Aussage Willi Sachses, der umfangreiche belastende Aussagen machte, ${ }^{133}$ und durch verfälschte Protokolle, die die Aussagen der Matrosen nicht richtig wiedergaben. ${ }^{134}$

Ein Musterbeispiel für die unter solchen Umständen zustande gekommenen Protokolle ist die Aussage Max Reichpietschs. Sie gibt in wesentlichen Teilen der USPD eine Mitverantwortung für die Vorkommnisse in der Hochseeflotte. ${ }^{135}$ Die Aussage von Albin Köbis stellt ebenfalls die Beteiligung der USPD heraus und verweist auf direkte Absprachen, ${ }^{136}$ die nie bestanden.

Mit diesen Aussagen konnte Dr. Dobring eine Anklage verfassen, die der USPD ein Programm unterstellte, das einen annexions- und kontributionslosen Frieden mit Hilfe des gewaltsamen Vorgehens der Matrosen herbeiführen sollte. ${ }^{137}$ Der Zettel Hans Beckers' vom 2. August 1917 mit der Erinnerung zur Solidarität war für Dr. Dobring der Beweis für die gewaltsamen Absichten der Flotte. ${ }^{138}$

125 Bernhard, Hans-Joachim: Unveröffentlichte Dokumente zum Aufstand in der deutschen Hochseeflotte im Sommer 1917, in: Zeitschrift für Geschichtswissenschaft 5 (1957), Dokument Nr. 2, Bericht der Politischen Polizei der Kriminalpolizei Kiel vom 2. September 1917, S. 1062.

126 Horn, Naval Mutinies, S. 138: "the prisoners still retained their confidence and optimism."

127 WUA, Bd. 9/II, S. 299.

128 Beckers, Zum Tode verurteilt, S. 45 - 47 und WUA, Bd. 9/II, S. 281.

129 WUA, Bd. 9/II, S. 281. Auch Beckers, Zum Tode verurteilt, S. 48.

130 Ebd., S. 252 und Beckers, Zum Tode verurteilt, S. 50.

131 Beckers, Zum Tode verurteilt, S. 48; Dittmann, Marine-Justizmorde, S. 44-45 und WUA, Bd. 9/II, S. 282.

132 WUA, Bd. 9/II, S. 342.

133 Ebd., S. 346 und WUA, Bd. 9/II, S. 380. Auch Horn, Naval Mutinies, S. 142.

134 Dittmann, Marine-Justizmorde, S. 44. Das bestätigten auch Beckers, WUA, Bd. 9/II, S. 282 und Weber, WUA, Bd. 9/II, S. 301-302.

135 WUA, Bd. 9/I, S. 297-299. Kürzere Fassung in BA-MA Freiburg, N 253-138, Nachlass von Tirpitz, Aussage Max Reichpietsch, 10. August 1917, Bl. 116-117.

136 BA-MA Freiburg, N 253 - 138, Nachlass von Tirpitz, Aussage Albin Köbis, 18. August 1917, Bl. 118 119.

137 WUA, Bd. 9/I, S. 61-62 und WUA, Bd. 9/II, S. 216. Auch Dittmann, Marine-Justizmorde, S. 55.

138 Ebd., Schriftliche Aussage Dr. Dobring, S. 490 und Beckers, Zum Tode verurteilt, S. 52. 
In dem Prozess selbst, der nur einen einzigen Verhandlungstag in Anspruch nahm, hatte die Anklage leichtes Spiel, zumal den Angeklagten nur sehr kurzfristig Pflichtverteidiger gestellt wurden, die kaum eine Möglichkeit besaßen, sich in die Akten einzuarbeiten. ${ }^{139}$

In dem Prozess verhielten sich die angeklagten Matrosen weitgehend zurückhaltend, da nach dem anfänglichen Geständnis Willi Sachses kaum eine wirkungsvolle Verteidigung möglich war. ${ }^{140}$ Albin Köbis begegnete dem Gericht mit Trotz und reagierte auf eine Zeugenaussage unbesonnen mit dem Ausruf »Wir sind Sozialrevolutionäre ${ }^{141}$. Das besiegelte sein Schicksal ebenso wie für Max Reichpietsch die Kontakte zur USPD. Dr. Dobring forderte für die Angeklagten Reichpietsch, Sachse und Weber die Todesstrafe. Für die Oberheizer Fischer und Bräuner beantragte der Marinehilfskriegsgerichtsrat 15 und 12 Jahre Zuchthaus. Dr. Breil beantragte für Hans Beckers und Albin Köbis ebenfalls die Todesstrafe. ${ }^{142}$ Zeitgleich mit der Untersuchungshaft und dem Prozess gegen die Matrosen informierte die Regierung führende Vertreter der Reichstagsparteien. In einer ersten Besprechung von Capelles mit Friedrich Ebert gab der Staatssekretär seine bisherigen Erkenntnisse an den SPD-Politiker weiter. Ebert war äußerst überrascht und empört über das Vorgehen der Matrosen, betonte aber, dass weder seine Partei noch die Unabhängigen Sozialdemokraten an den Vorfällen beteiligt sein könnten. ${ }^{143}$ In einer Konferenz des Reichskanzlers mit den Politikern der Reichstagsparteien verurteilte die Regierung die Matrosenbewegung und stellte ausdrücklich die Verantwortung der USPD heraus, gegen die strafrechtlich ermittelt werde. Reichskanzler Georg Michaelis hob hervor, dass bereits ein Todesurteil verhängt worden sei und weitere wahrscheinlich folgen würden. ${ }^{144}$ Mit Ausnahme des Konservativen Graf Westarp ${ }^{145}$ und des Abgeordneten der Deutschen Frak-

139 Dittmann, Marine-Justizmorde, S. 46. Hier ist aber die Leistung des Verteidigers Dr. Arkenau besonders herauszuheben, der sich für seinen Mandanten Albin Köbis über das zu erwartende Maß einsetzte und insbesondere die Anklage auf "vollendeten Aufstand" abstritt. Ein Verweis auf die Verurteilung Karl Liebknechts, der in Soldatenuniform öffentlich zur Desertion aufforderte und mit 54 Monaten Zuchthaus bestraft wurde, sollte das Strafmaß in diese Richtung abmildern. Siehe dazu Beckers, Zum Tode verurteilt, S. 70.

140 Horn, Naval Mutinies, S. 141-143.

141 Beckers, Zum Tode verurteilt, S. 62.

142 Ebd., S. $68-70$.

143 Dokumente und Materialien zur Geschichte der Deutschen Arbeiterbewegung, Reihe II, Bd. 1: Juli 1914 bis Oktober 1917, hrsg. vom Institut für Marxismus-Leninismus beim Zentralkomitee der Sozialistischen Einheitspartei Deutschlands, Berlin 1958, Dokument Nr. 230, Niederschrift über die Besprechung zwischen dem Staatssekretär des Reichsmarineamtes, von Capelle, und Ebert am 9. August 1917 über revolutionäre Unruhen in der Flotte, S. 657-658.

144 Admiral Scheer wandelte das Todesurteil am 3. September 1917 in eine vierjährige Gefängnisstrafe um. Siehe dazu Deist, Militär und Innenpolitik, Bd. 2, Dokument Nr. 383, Niederschrift über die Besprechung des Reichskanzlers mit führenden Abgeordneten der Reichstagsparteien, S. 1031.

145 Westarp, Kuno Graf: Konservative Politik im letzten Jahrzehnt des Kaiserreiches, Bd. 2: Von 1914 bis 1918, Berlin 1935, S. 625. 
tion Fritz Warmuth ${ }^{146}$ versuchten alle Politiker, zumindest die Vollstreckung der zu erwartenden Todesurteile zu verhindern. Sie verwiesen auf die zu erwartende innenpolitische Wirkung. ${ }^{147}$

Reichskanzler Michaelis wusste aber selbst, dass für eine Anklage keine belastbaren, juristisch verwertbaren Beweise vorlagen. ${ }^{148}$ Die ermittelnde Reichsanwaltschaft kam deshalb auch schnell zu dem Ergebnis, Abgeordnete der USPD strafrechtlich nicht belangen zu können. ${ }^{149}$ Auf Druck der Regierung ermittelte die Anwaltschaft weiter, ${ }^{150}$ leitete Verfahren gegen Luise Zietz und Emil Büdeler ein, ${ }^{151}$ die aber allesamt keine Beweise für eine Beteiligung der USPD an der Gehorsamsverweigerung in der Hochseeflotte erbrachten. ${ }^{152}$ Die Regierung Michaelis wollte die erfolglosen Ermittlungen nicht akzeptieren und beging in der Reichstagssitzung vom 9. Oktober mit der unbewiesenen Beschuldigung der USPD einen schweren politischen Fehler, der zum Sturz des Reichskanzlers führte.

Das zuerst ausgesprochene Todesurteil gegen Bernhard Spanderen verhängten die Richter wegen seiner maßgeblichen Rolle während des Matrosenausmarsches vom 1. August 1917. Das Gericht verurteilte ihn wegen erwiesener "verbrecherischer Energie» zum Tode. ${ }^{153}$ Wie willkürlich das Urteil war, zeigt sich an der vollkommen anderen Beurteilung durch Admiral Scheer, der die Bestätigung verweigerte und die Strafe auf vier Jahre Gefängnis minderte.154 Der sehr große Unterschied bei der Höhe des Strafmaßes verdeutlicht, wie unterschiedlich die Handlungen der Matrosen in der Marineleitung selbst betrachtet werden konnten.

Das gilt auch für die Todesurteile gegen Albin Köbis, Max Reichpietsch, Willi Weber, Willi Sachse und Hans Beckers wegen «vollendeter kriegsverräterischer Aufstandserregung «155. Genau an diesem entscheidenden Punkt gingen die Meinungen selbst der Marinejuristen auseinander. Das Gericht betrachtete den Ausmarsch der Matrosen vom 2. August als äußerst schwerwiegenden Tatbestand,

146 Warmuth vertrat den Wahlkreis Liegnitz. Er beteiligte sich 1918 an der Gründung der DNVP und trat 1933 in die NSDAP ein. Sein weiterer Weg und auch das Todesdatum sind nicht bekannt.

147 Deist, Militär und Innenpolitik, Bd. 2, Dokument Nr. 383, Niederschrift über die Besprechung des Reichskanzlers mit führenden Abgeordneten der Reichstagsparteien, S. 1038.

148 Michaelis, Georg: Für Staat und Volk. Eine Lebensgeschichte, Berlin 1922, S. 362.

149 WUA, Bd. 9/I, S. 87 und Westarp, Konservative Politik, Bd. 2, S. 625.

150 Bernhard, Aufstand in der Hochseeflotte, S. 210 - 212.

151 BA-MA Freiburg, RM 47-140, Gerichtsangelegenheiten 1917-1918, Beschluss des Oberreichsanwalts, Cöpenick 4. Oktober 1917, Bl. 33.

152 WUA, Bd. 9/I, S. 100.

153 WUA, Bd. 10/I, Urteil in der Untersuchungssache gegen Handgrödinger und Mitangeklagte, S. 320 321.

154 Ebd., Bestätigungsorder zum Urteil in der Untersuchungssache gegen Handgrödinger und Mitangeklagte vom 3. September 1917, S. 320.

155 Ebd., Urteil in der Untersuchungssache gegen Sachse, Reichpietsch und Mitangeklagte, S. 324. 
der das Todesurteil rechtfertigte. Dr. Felisch, Leiter der Justizabteilung im Reichsmarineamt, bewertete die Vorgänge in einem Gutachten vom 16. August anders, da ein Aufstand nicht vorgelegen habe. Eine Verurteilung könne höchstens auf der Grundlage eines versuchten Aufstandes erfolgen. ${ }^{156}$ Das schloss ein Todesurteil aus.

Dieser Sicht schloss sich nach Verhängung der Todesurteile der Gutachter de Bary an. Er hob hervor, dass die Todesurteile aufgrund einer vollendeten Aufstandserregung anfechtbar seien, da kein sichtbarer Aufstand stattgefunden habe. De Bary empfahl Admiral Scheer, die Todesurteile in lebenslange Haft umzuwandeln. ${ }^{157}$ Das Gericht hätte trotz gemildertem Strafmaß angesichts der immer noch strengen Strafe von lebenslanger Haft nicht an Ansehen eingebüßt. Admiral Scheer wäre aus der schwierigen Lage befreit gewesen, Todesurteile auf einer zumindest sehr fragwürdigen Rechtsgrundlage zu bestätigen. Obwohl er die Bedenken anerkannte, die auch von dem Prozessbeobachter Hauptmann Brandes schriftlich geäußert wurden, ${ }^{158}$ bestätigte er die Todesurteile gegen Max Reichpietsch und Albin Köbis. ${ }^{159}$

Vieles spricht dafür, dass zwei Todesurteile als abschreckendes Beispiel unbedingt vollstreckt werden sollten. Bereits vor der Urteilsverkündung organisierte Admiral Scheer die Erschießungen in Köln. ${ }^{160}$ Dieses Vorgehen beurteilte Dittmann als "militärischen Willkürakt aus politischen Motiven. «161 $\mathrm{Zu}$ dem gleichen Ergebnis gelangte auch Horn und bezeichnete das Urteil als "judical murder « ${ }^{162}$. Besonders der bewusst zurückgehaltene letzte Brief Max Reichpietschs, in dem er die Möglichkeit eines Gnadengesuchs an den Kaiser erwähnte, spricht für die Absicht, zwei Matrosen hinrichten zu lassen. ${ }^{163}$ Nach Hans Beckers waren die politischen Kontakte Max Reichpietschs grundlegend für die Bestätigung der Todesstrafe. ${ }^{164}$ Richard Müller spricht ausdrücklich von "Schreckensurteilen« ${ }^{165}$, die für

156 WUA, Bd. 9/I, S. 71. Auch bei Dittmann, Marine-Justizmorde, S. 62 - 63.

157 WUA, Bd. 9/I, S. 71.

158 Ebd., S. 73 - 74 und Dittmann, Marine-Justizmorde, S. 65. Hauptmann Brandes war im Sommer 1917 im Admiralstab tätig.

159 Ebd., S. 75 und Dittmann, Wilhelm: Erinnerungen, Bd. 3 (Quellen und Studien zur Sozialgeschichte, Bd. 14), bearbeitet und eingeleitet von Jürgen Rojahn, Frankfurt a. M./ New York 1995, S. 913.

160 WUA, Bd. 9/I, S. $74-75$ und Dittmann, Marine-Justizmorde, S. $65-66$.

161 Dittmann, Erinnerungen, Bd. 3, S. 915.

162 Horn, Naval Mutinies, S. 165.

163 BA-MA Freiburg, RM 20 - 112, Rechtsverhältnisse, Der Staatssekretär des Reichsmarine-Amtes an das Kommando der Hochseestreitkräfte durch Dr. Felisch, 20. November 1917, B1. 49 und BA-MA Freiburg, RM 20-112, Rechtsverhältnisse, Kommando des IV. Geschwaders an das Kommando der Hochseestreitkräfte, 23. November 1917, Bl. 51.

164 Beckers, Zum Tode verurteilt, S. 60. Bei Albin Köbis gab seine unnachgiebige Haltung den Ausschlag, das Todesurteil zu bestätigen. Siehe dazu Horn, Naval Mutinies, S. 164.

165 Müller, Richard: Geschichte der deutschen Revolution, Bd. 1, Vom Kaiserreich zur Republik (Kritische Bibliothek der Arbeiterbewegung, Bd. 3), Berlin 1979, S. 137. 
Empörung unter Mannschaften und Offizieren sorgten. ${ }^{166}$ Doch die Marinejustiz beschritt weiter den Weg strengster Urteile. In dem Prozess gegen die Besatzung der "Westfalen« verhängte sie vier Todesurteile gegen die Matrosen Michael Hiller, Hans Haase, Paul Brügge und ihren Kameraden Siegfried.167 Diese Urteile bestätigte Admiral Scheer, auch nach Protesten von Reichstagsabgeordneten, ${ }^{168}$ jedoch nicht. ${ }^{169}$

Wie unangemessen die Verurteilung wegen eines vollendeten Aufstandes war, zeigt sich deutlich bei einem Vergleich mit dem Vorgehen der österreichisch-ungarischen Matrosen in Cattaro vom Februar 1918. Gleich zu Beginn des Aufstandes bewaffneten sich die Matrosen. Ein Offizier wurde bei dem Versuch Widerstand zu leisten durch einen Kopfschuss lebensgefährlich verletzt. ${ }^{170}$ Nach diesem gewaltsamen Beginn zeigten sich die organisatorischen Defizite der Matrosen, die sich zwar gewaltsam gegen weitverbreitete Missstände empörten, aber den begonnenen Aufstand nicht zielgerichtet durchführen konnten. ${ }^{171}$ Durch eine umfassende Nachrichtensperre gelangten keine Informationen an mögliche politische oder militärische Unterstützer. ${ }^{172}$ So fiel es der Marineleitung nach wenigen Tagen verhältnismäßig leicht, die isolierten Matrosen zur Aufgabe zu bewegen. ${ }^{173}$ Die folgende juristische Aburteilung fiel sehr grob aus. Aufgrund von Zeugenaussagen belastete Matrosen galten als überführt und wurden in einem Schnellverfahren abgeurteilt und erschossen. ${ }^{174}$ Die individuelle Schuld war nicht nachgewiesen.

Der Vergleich mit den Vorfällen in Cattaro veranschaulicht die Gewaltfreiheit der deutschen Matrosenbewegung. Sie bewaffneten sich nicht und gefährdeten zu keinem Zeitpunkt die Kriegsbereitschaft der Hochseeflotte.

Die stürmisch verlaufende Reichstagssitzung vom 9. Oktober bildete den politischen Abschluss der Matrosenbewegung des Sommers 1917. Auf eine Anfrage Wilhelm Dittmanns nach den Todesurteilen für die Matrosen der Hochseeflotte ${ }^{175}$

166 WUA, Bd. 10/I, Tagebuch Richard Stumpf, S. 51.

167 BA Berlin, R 1501/112 474, Umtriebe in der Marine, Urteil gegen Haase und Genossen von der "Westfalen", Abschrift 23. November 1917, Bl. 400. Vorname Siegfrieds dort nicht angegeben. Auch WUA, Bd. 9/I, S. 51 und Horn, Naval Mutinies, S. 152.

168 Scheidemann, Philipp: Memoiren eines Sozialdemokraten, Bd. 2, Dresden 1930, S. 159-160.

169 BA Berlin, R 1501/112 474, Umtriebe in der Marine, Urteil gegen Haase und Genossen von der "Westfalen«, Abschrift 23. November 1917, Bl. 409.

170 Frei, Bruno: Die Matrosen von Cattaro. Eine Episode aus dem Revolutionsjahr 1918 (Gewehre in Arbeiterhand), Berlin 1963, S. 47.

171 Plaschka, Richard Georg: Cattaro - Prag. Revolte und Revolution. Kriegsmarine und Heer Österreich-Ungarns im Feuer der Aufstandsbewegungen vom 1. Februar und 28. Oktober 1918 (Veröffentlichungen der Arbeitsgemeinschaft Ost, Bd. 3), Graz/ Köln 1963, S. 97.

172 Karolyi, Graf Michael: Gegen eine ganze Welt. Mein Kampf um den Frieden, München 1924. S. $344-345$.

173 Plaschka, Cattaro - Prag, S. $173-180$.

174 Frei, Matrosen von Cattaro, S. $103 \mathrm{f}$.

175 Verhandlungen des Reichstages, Stenographische Berichte, Bd. 310, S. 37701. 
ging die Regierung in die Offensive und beschuldigte die USPD, eine maßgebliche Verantwortung für die Vorkommnisse zu tragen. ${ }^{176}$ Trotz der nicht vorhandenen Beweise griffen Reichskanzler Michaelis und sein Staatssekretär von Capelle eine Partei des Reichstages scharf an und trugen entscheidend dazu bei, dass sich die Mehrheitsparteien von Sozialdemokratie, ${ }^{177}$ Zentrum $^{178}$ und Liberalen ${ }^{179}$ für die USPD einsetzten und die Ankläger bloßstellen konnten. Damit war das ohnehin schon geringe Vertrauen der Mehrheitsparteien in den Reichskanzler vollkommen erschüttert. Georg Michaelis musste seinen Rücktritt einreichen.

Die historische Bewertung der Flottenbewegung des Sommers 1917 erfolgte zumeist in zwei Kategorien. In einer konservativen Tradition aus der Zeit der Weimarer Republik bis in die 1970er-Jahre der Bundesrepublik galten die Matrosen um Albin Köbis und Max Reichpietsch schlicht als Meuterer, die ein gerechtes Ende fanden. Admiral Brüninghaus sprach eindeutig von Meuterei und Kriegsverrat durch die Matrosen. ${ }^{180}$ Dr. Dobring sah in den Ereignissen den Vorläufer des Dolchstoßes, durch den die erfolgreiche Armee gemeuchelt wurde. ${ }^{181}$ Die Schrift „War es die Marine?« argumentiert ebenfalls in dieser Tradition und sieht die Schuld für die harten Urteile bei den Matrosen selbst. ${ }^{182}$ Heinrich Neu schloss sich diesem Muster weitgehend an und stellte die gesetzeswidrigen Taten der Matrosen heraus, für die Köbis und Reichpietsch "mit dem Tode sühnen" mussten. ${ }^{183}$ Noch in den 1970er Jahren wiederholte Legahn diese Argumentation. Die Matrosen hätten für ihre Meuterei aufgrund der eindeutigen Rechtslage nach einem rechtmäßigen Gerichtsverfahren die angemessene Strafe erhalten. ${ }^{184}$

Die sozialdemokratischen und sozialistischen Publikationen bewerteten die Matrosenbewegung hingegen vollkommen anders. Wilhelm Dittmann verfasste 1927 als SPD-Reichstagsabgeordneter eine grundlegende Schrift über das gesetzeswidrige Vorgehen der Marineleitung und der Militärjustiz, das er als "militärischen Willkürakt aus politischen Motiven« anprangerte. ${ }^{185}$ Diese Einschätzung

176 Ebd., S. 37751.

177 Ebd., S. 3775 r.

178 Ebd., S. 37891.

179 Ebd., S. 37951.

180 WUA, Bd. 9/I, S. 315.

181 Der Dolchstoß-Prozeß in München / Oktober-November 1925, Eine Ehrenrettung des deutschen Vol-

kes. Zeugen- u. Sachverständigen-Aussagen. Eine Sammlung von Dokumenten, München 1925, S. 60.

182 War es die Marine?, hrsg. vom Verein ehemaliger Matrosen der Kaiserlichen und der Reichsmarine von Erich Ehlert, Helmuth Krebs und Friedrich Will, Berlin 1926, S. 84 f.

183 Neu, Revolutionäre Bewegung, S. 38.

184 Legahn, Ernst: Meuterei in der Kaiserlichen Marine 1917/1918. Ursachen und Folgen (Koehlers kleine Seebücherei, Bd. 3), Herford 1970, S. 40.

185 Dittmann, Erinnerungen, Bd. 3, S. 915. 
bekräftigte der amerikanische Historiker Horn mit dem Begriff des »judical murder«. ${ }^{186}$

Die sozialistische Geschichtsschreibung beließ es nicht dabei, die Vorgehensweise der Reichsbehörden scharf zu kritisieren. Für sie waren gerade die hingerichteten Matrosen Vorkämpfer einer neuen Staatsform. Bereits der Spartakusbrief Nr. 7 vom November 1917 sah in Köbis und Reichpietsch »die wahren Helden des Weltkrieges«, die sich weigerten, weiterhin als Kanonenfutter für den Imperialismus herzuhalten. ${ }^{187}$

Für Luise Zietz galten die Matrosen bereits 1919 als Vorläufer der Revolution. ${ }^{188}$ Diese Argumentation führte die Geschichtsschreibung der Deutschen Demokratischen Republik fort. Die marxistischen Untersuchungen von Bernhard zur Flottenbewegung betonten das revolutionäre Potential der Bewegung, ${ }^{189}$ die durch "klassenbewußte Matrosen " getragen wurde. ${ }^{190}$ In dieser vorgegebenen Sichtweise unterstellten spätere Untersuchungen den Matrosen stets revolutionäre Absichten. ${ }^{191}$ Nach Horn diente diese Interpretation dem Versuch des noch jungen Staates, die Matrosenbewegung als Vorläufer des Sozialismus zu interpretieren. ${ }^{192}$

Sowohl die konservative als auch die sozialistische Interpretation der Flottenbewegung des Sommers blenden bewusst wichtige Tatsachen aus, um ihre Ergebnisse plausibel erscheinen zu lassen. Die konservative Richtung wollte die tatsächlichen Verhältnisse mit den entwürdigenden Bedingungen für die Matrosen nicht wahrhaben und übersah stets die Gewaltfreiheit der gesamten Bewegung bis zu ihrem Ende. Die sozialistische Geschichtsschreibung betonte immer wieder den revolutionären Charakter und die Nähe zum Spartakusbund. Tatsächlich standen selbst die herausragendsten Vertreter der Matrosenbewegung der Gruppe um Karl Liebknecht und Rosa Luxemburg fern. Viele von ihnen kannten die Schriften Lenins nicht. Gegen eine gezielte politische Ausrichtung der Matrosenbewegung spricht auch, dass es Albin Köbis und Hans Beckers strikt ablehnten, überhaupt zu einer Partei oder Gruppierung Kontakt aufzunehmen.

186 Horn, Naval Mutinies, S. 165.

187 Spartakusbriefe. Hrsg. vom Institut für Marxismus-Leninismus beim Zentralkomitee der Sozialistischen Einheitspartei Deutschlands, Berlin 1958, Nr. 7 vom November 1917, S. 403.

188 WUA, Bd. 9/I, S. 307.

189 Die Bezeichnung als »organisierte revolutionäre Mannschaftsbewegung«, Bernhard, Aufstand in der Hochseeflotte, S. 48, ist ideologisch geprägt und geht an der Realität vorbei. Siehe auch Bernhard, Revolutionäre Friedensbewegung, S. 89-140.

190 Bernhard, Aufstand in der Hochseeflotte, S. 4.

191 Gutsche, Willibald/ Klein, Fritz/ Petzold, Joachim: Von Sarajevo nach Versailles. Deutschland im ersten Weltkrieg, Berlin 1974, S. 203.

192 Horn, Naval Mutinies, S. XI: „In East Germany the new communist government and its historians sought to establish credentials for ruling by claiming the spirited proletarians in the World War I navy as the ancestors of their regime». 
Einen möglichen Weg zur historischen Einordnung bietet die von bundesdeutschen Marineoffizieren seinerzeit heftigst angefeindete ${ }^{193}$ Interpretation des Offiziers Dr. Friedrich Beermann. Der damalige Oberst und spätere Brigadegeneral unternahm 1958 den Versuch, die Matrosen in die Tradition der deutschen Marine einzugliedern: »Unser Bundestag hat diese Tatsache wohl erkannt und deswegen gesetzlich festgelegt, daß der Gehorsam sich bricht, wenn der Befehl die Menschenwürde verletzt. Steht unsere Marine aber innerlich auf dem politischen Boden, auf dem diese Gesetze gewachsen sind, so sollte sie auch jenen beiden Seeleuten ihren Respekt nicht versagen, die durch die Fehler eines vergangenen Militärsystems zu Symbolen vieler in ihrer Menschenwürde tief verletzter Soldaten und Matrosen geworden sind. Sie täte gut daran, diese aus der Kaiserlichen Marine verstoßenen Matrosen wieder in die große Familie jener aufzunehmen, die das blaue Tuch trugen.«194

Mit dem Ansatz Friedrich Beermanns wird das Handeln der Matrosen verständlich. Sie reagierten auf die unwürdige Behandlung eines autoritären Staates in Kriegszeiten. Ihr Bestreben war es, für ihre Menschenwürde zu kämpfen und durch eine Gehorsamsverweigerung der Flotte politische Schritte für einen Friedensschluss zu beschleunigen.

Da die Matrosen sich gegen die Anwendung von Gewalt aussprachen und nie zu den Waffen griffen, kann die Bewegung des Sommers 1917 als Bemühung gesehen werden, die ihnen verweigerte Würde einzufordern. Das höchste Ziel der Matrosen bestand darin, durch das eigene Handeln ein Zeichen zu setzen, dass sie ebenso wie die Bevölkerung des Krieges überdrüssig waren und nur noch Frieden wollten.

Die zentrale Fragestellung der Ringvorlesung "Die große Furcht» kann für die Geschichte der deutschen Matrosenbewegung des Sommers 1917 kurz beantwortet werden. Die Offiziere und Admirale hatten keine Angst vor den Matrosen. Sie sahen stets auf die unteren Dienstränge herab und beschränkten den Kontakt auf das Nötigste. Sie sahen in den einfachen Soldaten Menschen, die zu gehorchen hatten. Sollten sie den Gehorsam wider Erwarten nicht in dem erforderlichen Maße erbringen, schritt die Marine-Justiz ein. Sie stellte zur Not auch durch Todesurteile die erforderliche Disziplin wieder her. Damit glaubte die Admiralität über genügend Zwangsmaßnahmen zu verfügen, um stets Herr der Lage sein zu können. Diese Einstellung behielt sie bis in die bereits ausbrechende Revolution

193 Nach seinem Vortrag herrschte eisiges Schweigen, nach einer kurzen Pause verlas der Rangälteste, Fregattenkapitän Schuhart, eine Erklärung, die sich von Köbis und Reichpietsch als zu Recht verurteilte Meuterer distanzierte. Nach einer Entgegnung Beermanns kam es zum Eklat, die Offiziere verließen den Saal. Ausführlicher Bericht in: Der Spiegel, 49/1958.

194 BA-MA Freiburg, N 597-190, Nachlass Dr. Friedrich Beermann, Vorträge, Bl. 34 - 35. 


\section{| Christoph Regulski}

bei. Erst als sich die Matrosen bewaffneten und der juristischen Gewalt etwas entgegensetzen konnten, war es mit der Macht der Offiziere vorbei. 